\title{
Association of serum gamma glutamyltransferase with diabetes mellitus, random blood sugar and body mass index in selected Sri Lankan subjects
}

\author{
Devika Bandara ${ }^{1}$, Sachini Thennakoon ${ }^{1}$, Pathiranage Dias², Niroshima Withanage ${ }^{1}$ \\ ${ }^{1}$ Department of Medical Laboratory Sciences, Faculty of Allied Health Sciences, University of Sri Jayewardenepura, Nugegoda, \\ Sri Lanka \\ ${ }^{2}$ Department of Statistics, Faculty of Applied Sciences, University of Sri Jayewardenepura, Nugegoda, Sri Lanka
}

\author{
Article Information \\ Total number of \\ Words 1201 \\ Tables 2 \\ Figures 2 \\ Authors have no conflicts of interest to \\ declare \\ Keywords: Diabetes mellitus, \\ Gamma glutamyltransferase, Body mass \\ index, Random blood sugar \\ Date of submission: 2020-07-07 \\ Date of acceptance: 2021-05-04 \\ Author responsible for correspondence: \\ Niroshima Withanage \\ Department of Medical Laboratory Sciences, \\ Faculty of Allied Health Sciences, University \\ of Sri Jayewardenepura, Nugegoda, Sri Lanka. \\ Email: withanagend@sjp.ac.lk
}

DOI: http://doi.org/10.4038/cjms.v57i1.4970

iD https://orcid.org/0000-0002-4602-5006

\begin{abstract}
Background

Serum gamma glutamyltransferase (GGT) have shown a strong association with diabetes mellitus (DM) in many reported studies. Studies have proven the association of elevated body mass index (BMI) and deposition of fat causing insulin resistance leading to increased serum GGT levels. Evidence is less supportive in the Sri Lankan context.
\end{abstract}

\section{Objectives}

The present study assessed the association of serum GGT with random blood glucose (RBS) and BMI in selected individuals with diabetes and individuals without diabetes.

\section{Methods}

Hundred and fourty-seven subjects including healthy individuals ( $\mathrm{n}=72$ individuals without diabetes) and individuals with type 2 diabetes $(n=75)$ were recruited by convenience sampling. Serum GGT, RBS and BMI were measured in all participants.

\section{Results}

The cases (median GGT=21.1 U/L, interquartile range $(\mathrm{IQR})=12.7-28.8 \mathrm{U} / \mathrm{L}$ ) showed a significantly elevated $(\mathrm{p}=0.024)$ median serum GGT level compared to controls (median GGT=14.4 U/L, IQR=12.3-20.1 U/L). There was a weak, positive but statistically significant correlation $(\mathrm{p}=0.001)$ between serum GGT and RBS in individuals with diabetes. The median serum GGT was significantly different $(\mathrm{p}=0.03)$ among the categories of BMI in cases. However, overweight group had a higher GGT (median GGT=24.1 U/L, $\mathrm{IQR}=14.6-32.0 \mathrm{U} / \mathrm{L}$ ) than the rest of the BMI categories in cases, while obese cases have a low serum GGT level (median GGT=19.6 U/L, IQR=12.6$26.9 \mathrm{U} / \mathrm{L})$ compared to overweight individuals.

\section{Conclusion}

Diabetes patients have significantly higher serum GGT levels compared to controls. Serum GGT levels positively associated with DM in the selected population with diabetes. Higher serum GGT levels were associated higher BMI. Serum GGT among BMI categories in cases showed a significant difference. 


\section{Background}

Serum gamma-glutamyltransferase (GGT) is a well-known important index of liver dysfunction and also a biological marker of alcoholic liver damage [1,2,3]. Physiological role of GGT enzyme is to counteract oxidative stress [4]. In addition, recent studies suggested strong associations of elevated GGT at the baseline and the risk of incidence of many cardiovascular diseases and metabolic syndrome. Many reported studies have shown a strong positive relationship between elevated serum GGT and diabetes mellitus (DM) [5-7]. Serum GGT level is associated with several other factors such as gender, advanced age, smoking, alcohol consumption, hypertension, lack of physical exercises, disorders of lipid metabolism and glycaemic disorders.

Overweight and obesity is increasing alarmingly in the developed and developing countries. Obesity is strongly associated with elevated GGT and excess fat deposition of liver leading to non-alcoholic fatty liver disease (NAFLD), which in turn causes hepatic insulin resistance and hyperinsulinaemia. Therefore, changes in GGT level reflects alterations of metabolic functions and could be used as a marker of insulin resistance [8,9]. Although mechanisms of the relationship between elevated GGT levels and insulin resistance are not clearly unders-tood, changes in GGT activity associated with diabetes is believed to be based on glutathione depletion and on the protective function of GGT induction to maintain appropriate hepatic glutathione levels [4]. Population based studies on the association of elevated GGT and metabolic syndrome are limited. Moreover, there is only limited evidence in the Sri Lankan context to support the association between serum GGT with blood glucose levels and BMI in individuals with diabetes. The present study investigated the association of serum GGT with random blood glucose (RBS) and body mass index in selected individuals with diabetes and individuals without diabetes.

\section{Methods}

\section{Study design}

This is an unmatched case-control study. Participants were recruited by convenience sampling. Cases were individuals with type 2 diabetes attending selected private laboratories and the controls (individuals without diabetes) were selected from the general population in a few selected districts in Sri Lanka.

\section{Study population}

A case control study was conducted including patients with type 2 diabetes (case) and apparently healthy individuals without diabetes (controls) between 20-60 years of age. The participants were recruited through a self-administered questionnaire. Previously confirmed individuals with type 2 diabetes were selected as cases after analyzing the questionnaire. All participants were screened for the exclusion criteria and written informed consent was obtained by all participants prior to the study. We excluded subjects with any previously diagnosed history of hepatobiliary diseases, NAFLD, subjects having hypertension, dyslipidaemia and subjects consuming alcohol.

\section{Study setting}

The study was conducted in the Faculty of Allied Health Sciences, University of Sri Jayewardenepura, Sri Lanka. Ethical approval was granted by the Ethics Review Committee of the University of Sri Jayewardenepura, Sri Lanka.

\section{Sample size calculation}

Sample size was calculated according to the two-sample t-test calculation criteria. Minitab version 16 software calculator was used for the calculation and calculated minimum sample size was 57 in each category of study subjects.

\section{Biological sample collection}

Five milliliters of non-fasting whole blood was collected by venepuncture and blood samples were collected into separate tubes according to the analysis. Blood tubes were labeled by the respective serial code given at the time of enrollment. Samples were centrifuged at $3000 \mathrm{rpm}$ for 5 minutes for serum separation, after keeping for 1 hour for clot formation they were stored at $4{ }^{\circ} \mathrm{C}$ prior to analysis. Further, serum samples were examined for hemolysis. Both random blood glucose (RBS) and serum GGT were measured by using a semi-automated clinical analyzer.

For all study participants body mass index (BMI) was calculated as (weight/height ${ }^{2}$ ). The BMI cut off values of $23-24.9 \mathrm{~kg} / \mathrm{m}^{2}$ and $\geq 25 \mathrm{~kg} / \mathrm{m}^{2}$ was considered to classify overweight and obesity respectively [10].

\section{Statistical analysis}

Analysis was done by SPSS version 25.0. General characteristics were analysed using descriptive analysis. Non-parametric tests were used for the analysis, as the serum GGT distribution deviated from normal distribution in both control and case groups.

Independent-Samples Mann-Whitney U test was used to compare the distribution of serum GGT across the control and case groups. Pearson's correlation 2-tailed test was performed with serum GGT and RBS levels and with serum GGT and BMI. The value of "r" was calculated in control (individuals without diabetes) and case (individuals with type 2 diabetes) respectively. 


\section{Results}

\section{Demographic characteristics of the study population}

The study was conducted using 147 individuals between 20-60 years of age. Out of 147 individuals 72 were individuals without diabetes (control group) (48.9\%) and 75 were known individuals with type 2 diabetes (case group) (51.0\%). Mean age of cases and controls were $53.7 \pm 5.7$ years and $33.8 \pm 14.3$ years $(\mathrm{p}<0.001)$ respectively. In the control group, majority were females $(77.8 \%)$ while males represented $22.2 \%$. There were $66.7 \%$ females and $33.3 \%$ males $(\mathrm{p}=0.133)$ in case group. Most of the control subjects were in normal BMI category (36.1\%) whereas in case subjects equal participants were found in overweight and obese BMI categories (33.3\%, $\mathrm{p}=0.066$ ). Cases showed non-significant ( $\mathrm{p}=0.066)$ elevated mean BMI $(24.2 \pm 4.2$ $\left.\mathrm{kg} / \mathrm{m}^{2}\right)$ compared to control subjects $\left(22.8 \pm 4.6 \mathrm{~kg} / \mathrm{m}^{2}\right)$ (Table 1).

\section{Distribution of serum GGT in the study population}

Cases showed significantly increased $(\mathrm{p}=0.024)$ GGT level (median GGT=21.1 U/L, IQR=12.7-28.8 U/L) compared to controls (median GGT=14.4 U/L, IQR=12.3-20.1 U/L). The majority of cases (86.7\%) and controls (88.9\%) showed serum GGT levels within normal reference range. However, there were 4 subjects ( $5.3 \%$ ) with elevated levels of GGT in case group while only $1.4 \%$ control subjects presented with elevated GGT levels. There was a significant difference between high, normal and low GGT levels (categorized according to the normal reference range of serum GGT $11.00-61.00 \mathrm{U} / \mathrm{L}$, reagent kit manual) in both cases $(\mathrm{p}<0.001)$ and controls $(\mathrm{p}<0.001)$.

\section{Association of serum GGT with RBS in study population}

According to the Pearson's correlation two tailed analysis, cases showed a weak, positive, but statistically significant correlation ( $\mathrm{r}=0.38$, $\mathrm{p}=0.001)$ between RBS and GGT levels (Figure $1(\mathrm{~A})$ ) while controls did not show a significant correlation between GGT and RBS (Figure 1 (B)).

\section{Association of serum GGT with BMI categories}

Cases showed an elevated level of serum GGT in overweight category (median=24.1 U/L, IQR=14.6-32.0 U/L) compared to that of control overweight category (median=15.6 U/L, IQR=12.6-21.0 U/L). The median serum GGT values were significantly different $(\mathrm{p}=0.032)$ among the categories of BMI (underweight, normal, overweight and obese) in individuals with diabetes. However, overweight group had a higher GGT (24.1 U/L) than the rest of the BMI categories in cases while obese cases had a low serum GGT level (median=19.6 U/L, IQR=12.6-26.9 $\mathrm{U} / \mathrm{L}$ ) compared to overweight individuals (Table 2). However, no significant difference in the distribution of serum GGT across the categories of BMI in the control group could be observed (Table 2).

Table 1. Distribution of socio-demographic variables among cases and controls

\begin{tabular}{llll}
\hline Variable & $\begin{array}{l}\text { Cases } \\
(\mathbf{n = 7 5 )}\end{array}$ & $\begin{array}{l}\text { Controls } \\
(\mathbf{n}=72)\end{array}$ & p value \\
\hline Age (years) (mean \pm SD) & $53.7 \pm 5.7$ & $33.8 \pm 14.3$ & 0.001 \\
Gender & $50(66.7 \%)$ & $56(77.8 \%)$ & 0.133 \\
Female (n, \%) & $25(33.3 \%)$ & $16(22.2 \%)$ & 0.066 \\
Male (n, \%) & $24.2 \pm 4.2$ & $22.8 \pm 4.6$ & $0.024 *$ \\
BMI (kg/m²) (mean \pm SD) & 21.1 & 14.4 & $\mathrm{p}<0.001$ \\
Median GGT (U/L) & $12.7-28.8$ & $12.3-20.1$ & \\
Interquartile range (IQR, U/L) & 178.2 & 95.7 & $84.9-113.7$ \\
Median RBS (mg/dL) & $121.6-254.9$ & & \\
IQR (U/L) & & & \\
\hline
\end{tabular}

${ }^{*} \mathrm{p} \leq 0.05$ considered as significant 


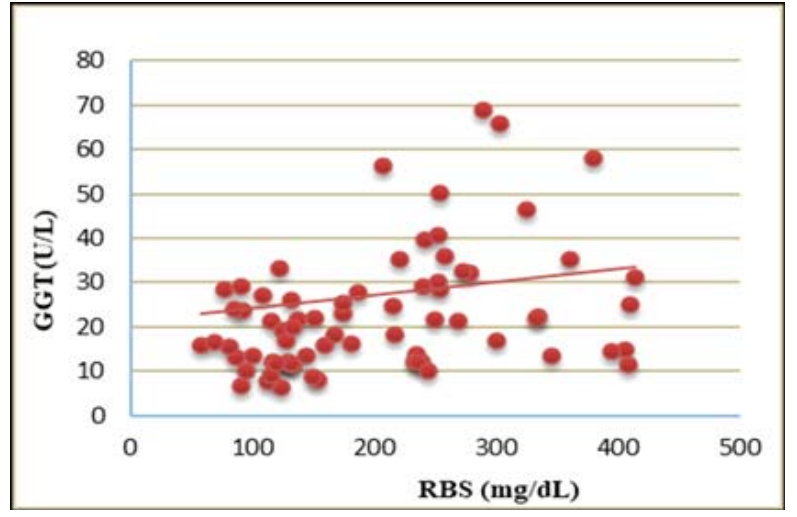

Figure 1(A). Distribution of serum GGT with RBS in the case group.

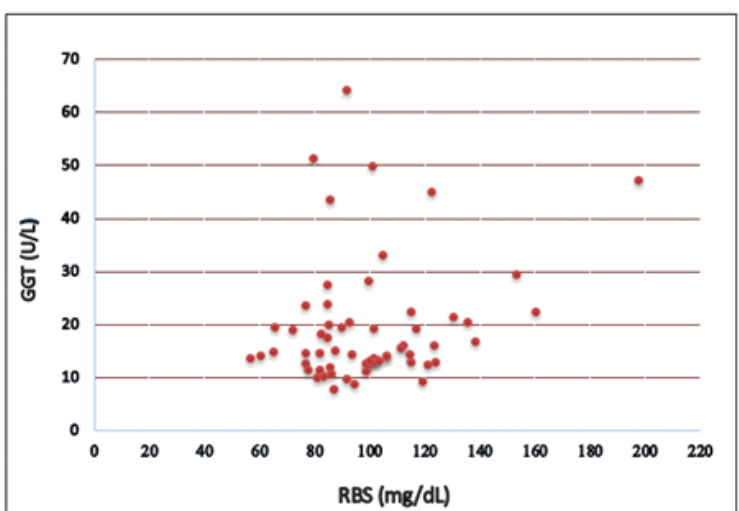

Figure 1(B). Distribution of serum GGT with RBS in the control group.

Table 2. Association of serum GGT with BMI categories

\begin{tabular}{|c|c|c|c|c|c|c|}
\hline \multirow[t]{2}{*}{ BMI Category } & \multicolumn{3}{|c|}{ Cases } & \multicolumn{3}{|c|}{ Controls } \\
\hline & n (\%) & $\begin{array}{l}\text { Median } \\
\text { Serum } \\
\text { GGT (U/L), } \\
\text { IQR(U/L) }\end{array}$ & p value & n (\%) & $\begin{array}{l}\text { Median } \\
\text { Serum } \\
\text { GGT(U/L), } \\
\text { IQR(U/L) }\end{array}$ & $p$ value \\
\hline Under weight & $4(5.3)$ & $\begin{array}{l}10.9 \\
9.1-14.9\end{array}$ & $0.03 *$ & 13 (18.1) & $\begin{array}{l}13.1 \\
11.3-18.8\end{array}$ & 0.48 \\
\hline Normal weight & $21(28.0)$ & $\begin{array}{l}16.7 \\
12.0-27.2\end{array}$ & & $26(36.1)$ & $\begin{array}{l}14.3 \\
11.9-21.7\end{array}$ & \\
\hline Overweight & 25 (33.3) & $\begin{array}{l}24.1 \\
14.6-32.0\end{array}$ & & 14 (19.4) & $\begin{array}{l}15.6 \\
12.6-21.0\end{array}$ & \\
\hline Obese & 25 (33.3) & $\begin{array}{l}19.6 \\
12.6-26.9\end{array}$ & & $19(26.4)$ & $\begin{array}{l}14.8 \\
12.9-24.3\end{array}$ & \\
\hline
\end{tabular}

${ }^{*} \mathrm{p} \leq 0.05$ considered as significant

\section{Discussion}

The present study was conducted to investigate the association of serum GGT with RBS and BMI in a selected Sri Lankan population. In this study 147 subjects were recruited. The control group consisted of 72 apparently healthy individuals without diabetes and case group consisted of 75 individuals with type 2 diabetes. Cases showed significantly increased GGT level compared to controls. According to the results it was found that the individuals with diabetes (median=21.1 U/L, IQR=12.728.8 U/L) had significantly higher $(\mathrm{p}=0.024)$ serum GGT levels compared to individuals without diabetes (median=14.4 U/L, IQR=12.3-20.1 U/L). Our results are consistent with several other reported studies [11-19]. Umeki et. al., (1989) recorded significantly elevated serum GGT in subjects with DM while a study carried out by H. Sakugawa et. al., (2004) stated that GGT elevation was independently associated with DM $[8,20]$. It is believed that contribution of GGT to maintain oxidative stress, insulin resistance and hepatic inflam-mation which impairs insulin signalling in the liver and other oragns determines the possible association of serum GGT and DM [2,8,21].

Although we included apparently healthy individuals in the control group, 7 subjects had a lower serum GGT value 
and one subject had a higher value than the normal reference range. Therefore, only 64 subjects had their serum GGT levels within the normal reference range. Among the cases 65 subjects had their serum GGT levels within the normal reference range. However, 6 subjects had a lower value and 4 subjects had a higher value than the normal reference range. When analyzing the distribution of serum GGT within the study population, we found two exceptional cases among the case group where they showed comparatively high serum GGT levels (164.4 U/L, 190.5 U/L) which deviate from the pattern. However, we could not find a reasonable explanation for their deviation. A proper clinical follow-up should be carried out in these instances to find out the exact cause for the abnormal elevation.

We obtained a significant positive but a weak correlation between serum GGT and RBS in individuals with type 2 diabetes. In the literature, there is no evidence for correlation between serum GGT and random blood glucose level, but evidence is present for the association of serum GGT with fasting blood glucose. Fraser et al., (2007) reported that GGT was associated with fasting glucose in women with diabetes and non-diabetes women, and the association between GGT and fasting glucose was stronger in women with diabetes than in women without diabetes. Authors also stated that association of GGT with glucose was similar among obese and non-obese nondiabetes women [5]. In the univariate analysis conducted by H. Sakugawa et al., (2004) serum GGT level correlated with blood glucose level [8]. Several other studies also also had a positive association with serum GGT and fasting blood glucose level $[2,8,17,19]$, thus strengthening the present study findings.

We excluded subjects specially with any previously diagnosed history of hepatobiliary diseases and NAFLD, as increased levels of GGT could be observed in those patients resulting falsely higher GGT levels other than diabetes. However, NAFLD may present undiagnosed in the general population which act as a limitation in the present study. For controls we excluded subjects having hyper-tension, dyslipidaemias, alcohol intake and DM as those will cause increase GGT levels which might affect the final results.

A reason for elevated GGT in individuals with diabetes in our study could be due to insulin resistance which is a major feature of type 2 diabetes. Due to insluin resistance, there can be excess deposition of fat in liver as a result of either increased production or mobilization of free fatty acids or decreased hepatic clearance of free fatty acids [22]. Other than diabetes mellitus, fatty change in liver can result from obesity, alcohol, hepatitis and various drugs [23]. Diabetes is a pathological condition where vascular reactive oxygen species production is increased. As a result of both increased fatty acids in liver and increased production of vascular reactive oxygen species, oxidative stress within hepatocytes is increased leading to decreased extra cellular glutathione and hepatocellular necrosis. Decreased glutathione along with cell turnover and cellular stresses would cause GGT to be leaked into serum resulting elevated serum GGT levels [2].

However, we did not conduct any investigation related to fatty liver which remians as a major limitation of the study. Although, the present study did not find significant correlation between serum GGT level and BMI, some studies have observed contrasting findings to the present study [3, 8, 11,17-19] .

Present study did not consider age and sex matched casecontrol subjects, which could be the major reason for not obtaining a correlation between serum GGT and BMI as serum GGT level is known to be affected by both age and sex. In the present study, both groups had a lower male population and most of the subjects in the diabetes population were above 35 years of age $(p<0.001)$.

Since average GGT values are significantly different for males and females, the data were analyzed separately for males and females. Age and the BMI were used as covariates (to remove the effect of age and BMI statistically) in comparing means of GGT for cases and controls. For males, it is evident that there is no significant difference ( $\mathrm{p}=0.888$ ) between means of GGT for cases and controls even if the age and BMI were the same. For females, it is evident that there is a significant difference $(\mathrm{p}=0.043$ ) between means of GGT for cases and controls if the age and BMI were the same, at $5 \%$ level of significance. Though average BMI values are different for cases and controls, the average difference in GGT is not due to the changes in BMI. It is interesting that both age and BMI are not significant in the model. Only the group (control/ case) is significant.

Another limitation was consideration of a single measurement of serum GGT and RBS which limits the precision of both values. Further, glycated haemoglobin, which is a valuable laboratory diagnostic indicator to monitor long term diabetes (over three months) could be a better indicator over RBS measurement which could be considered as a limitation.

According to the statistical analysis, serum GGT was significantly different across the categories of BMI in individuals with type 2 diabetes (case group). The overweight subjects had significantly higher serum GGT levels compared to underweight individuals with diabetes. However, in the present study we did not get a trend of increasing serum GGT levels with BMI category. Similar findings were reported in a study conducted in Norwegian men, which showed progressively increased GGT across 
all classes of BMI from $<20 \mathrm{~kg} / \mathrm{m}^{2}$ to $>35 \mathrm{~kg} / \mathrm{m}^{2}$ [24]. However, the BMI cut off points used in this study deviate from our Asian BMI cut off points.

Although, several several studies have reported the association of serum GGT with blood glucose level and BMI elsewhere, in the Sri Lankan context evidence is limited to the best of our knowledge which remains as a major strength of the present study. The authors recommend to conduct a follow-up study to observe the association between serum GGT and development of type 2 diabetes on a pre-diabetes population and further studies can be conducted to evalaute the progression of NAFLD and cirrohosis in individuals with type 2 diabetes. Moreover, studies should be designed to evaluate the association of higher GGT with cardiovascular risk in individuals with type 2 diabetes.

\section{Conclusion}

Individuals with type 2 diabetes have significantly higher serum GGT levels compared to appenrently healthy individuals without diabetes. Higher RBS is associated with higher GGT among individuals with diabetes. There was a trend of increasing GGT with increasing BMI in individuals with type 2 diabetes, but not in the obese group. However, there was no such association between GGT and RBS or BMI among individuals without diabetes. If similar associations could be proved in a national cluster in future studies, serum GGT could be served as a risk marker estimate for diabetes mellitus.

\section{Author's contributions}

DB, ST and NW conceived the study concept. Experimental studies and data collection and data analysis were conducted by DB and ST. First draft of the manuscript was prepared by DB and ST, while final editing was done by NW.

\section{References}

1. Kunustor SK, Abbasi A, Amanda IA, Gamma-glutamyl transferase and risk of type II diabetes: an updated systemic review and dose response meta-analysis. Annals of Epidemiology 24(2014); 809-816.

doi: 10.1016/j.annepidem.2014.09.001

2. Lee DH, Jacobs DR, Gross M, et al. $\gamma$-glutamyltransferase is a predictor of incident diabetes and hypertension: the Coronary Artery Risk Development in Young Adults (CARDIA) Study. Clinical Chemistry. 2003; 49(8): 1358-66. doi: $10.1373 / 49.8 .1358$

3. Lee DS, Evans JC, Robins SJ, et al. Gamma glutamyl transferase and metabolic syndrome, cardiovascular disease, and mortality risk: the Framingham Heart Study. Arteriosclerosis, Thrombosis, and Vascular Biology. 2007; 27(1): 127-133.

doi: 10.1161/01.ATV.0000251993.20372.40
4. Whitfield J. Gamma glutamyl transferase. Critical Reviews in Clinical Laboratory Sciences 2001; 38(4): 263-355.

5. Fraser A, Ebrahim S, Davey Smith G, et al. A comparison of associations of alanine aminotransferase and gammaglutamyltransferase with fasting glucose, fasting insulin, and glycated hemoglobin in women with and without diabetes. Hepatology 2007; 46(1): 158-65. doi: 10.1002/hep.21667

6. Doi Y, Kubo M, Yonemoto K, Ninomiya T, Iwase M, Tanizaki Y, Shikata K, Lida M, Kiyohara Y. Liver enzymes as a predictor for incident diabetes in a Japanese population: the Hisayama study. Obesity 2007; 15(7): 1841-50.

7. Balogun W, Adeleye J, Akinlade K, Adedapo K, Kuti M. Frequent occurrence of high gamma-glutamyl transferase and alanine amino transferase among Nigerian patients with type 2 diabetes. African Journal of Medicine and Medical Sciences. 2008; 37(2): 177-83.

8. Sakugawa H, Nakayoshi T, Kobashigawa K, et al. Metabolic syndrome is directly associated with gamma glutamyl transpeptidase elevation in Japanese women. World Journal of Gastroenterology 2004Apr1; 10(7): 1052. doi: 10.3748/wjg.v10.i7.1052

9. Meisinger C, Löwel H, Heier M, et al. KORA Study Group. Serum $\gamma$ glutamyltransferase and risk of type 2 diabetes mellitus in men and women from the general population. Journal of Internal Medicine. 2005 Dec; 258(6): 527-35. doi: 10.1111/j.1365-2796.2005.01572.x

10. James WP, Chunming C, Inoue S. Appropriate Asian body mass indices?. Obesity Reviews 2002 Aug; 3(3): 139. doi: 10.1046/j.1467-789x.2002.00063.x.

11. Barbieux J, Bacq Y, Schellenberg F, et al. Increase of serum gamma-glutamyl transferase activity in diabetic patients is not linked to diabetes itself. Pathologie-biologie. 1990; 38(2): 93-98.

12. Orrell JM, Neithercut WD, Henderson J, et al. Raised liver associated enzyme activity and post-prandial bile acid concentrations in sera from treated diabetic outpatients. Diabetes Research and Clinical Practice 1990; 10(1): 51-57. doi:10.1016/0168-8227(90)90081-4

13. André P, Balkau B, Vol S, et al. $\gamma$-glutamyltransferase activity and development of the metabolic syndrome (International diabetes federation definition) in middle-aged men and women: data from the epidemiological study on the insulin resistance syndrome cohort. Diabetes Care 2007; 30(9): 2355-61. doi: $10.2337 /$ dc07-0440

14. Luxmi S, Sattar RA, Ara J. Association of non-alcoholic fatty liver with type 2 diabetes mellitus. Journal of Liaquat University of Medical and Health Sciences 2008; 9: 188-93.

15. Forlani G, Di Bonito P, Mannucci E, et al. Prevalence of elevated liver enzymes in Type 2 diabetes mellitus and its association with the metabolic syndrome. Journal of Endocrinological Investigation 2008; 31(2): 146-52.

16. Colloredo-Mels G, Bettale G, Bellati G, et al. Gammaglutamyl-transpeptidase in diabetics: a case control study. Clinica Chimica Acta. 1988; 175(2): 189-95.

doi: 10.1016/0009-8981(88)90009-5 
17. Brenner H, Rothenbacher D, Arndt V, et al. Distribution, determinants, and prognostic value of $\gamma$-glutamyltransferase for all-cause mortality in a cohort of construction workers from southern Germany. Preventive Medicine 1997; 26(3): 305-10. doi: 10.1006/pmed.1997.0144

18. Wannamethee G, Ebrahim S, Gerald Shaper A. Gammaglutamyltransferase: determinants and association with mortality from ischemic heart disease and all causes. American Journal of Epidemiology 1995; 142(7): 699-708. doi: 10.1093/oxfordjournals.aje.a117699

19. Emdin M, Pompella A, Paolicchi A. Gamma-glutamy ltransferase, atherosclerosis, and cardiovascular disease: triggering oxidative stress within the plaque. American Heart Association; 2005.

doi: 10.1161/CIRCULATIONAHA.105.571919

20. Umeki S, Hisamoto N, Hara Y. Study on background factors associated with impaired glucose tolerance and/or diabetes mellitus. European Journal of Endocrinology 1989; 120(6): 729-34.

doi: 10.1530/acta.0.1200729

21. Sabanayagam C, Shankar A, Li J, et al. Serum gammaglutamyl transferase level and diabetes mellitus among US adults. European Journal of Epidemiology 2009; 1: 24(7): 369-73.

22. Fernández-Real JM, López-Bermejo A, Ricart W. Crosstalk between iron metabolism and diabetes. Diabetes 2002; 51(8): 2348-54.

doi: $10.2337 /$ diabetes.51.8.2348

23. Neuschwander-Tetri BA. NASH. Zakim and Boyer's Hepatology: Elsevier; 2006. 1031-63.

24. Robinson D, Whitehead T. Effect of body mass and other factors on serum liver enzyme levels in men attending for well population screening. Annals of Clinical Biochemistry 1989; 26(5): 393-400. doi: $10.1177 / 000456328902600503$ 